\title{
Visual motor integration delay in preschool children infected with HIV
}

\author{
Ramona Odejayi, BSc OT (Wits); MSc OT (Wits) http://orcid.org/0000-0002-3353-766X \\ Occupational therapist, Northcott, Sydney, Australia \\ Postgraduate student, Department of Occupational Therapy, School of Therapeutic Sciences, Faculty of Health Sciences, \\ University of the Witwatersrand
}

Denise Franzsen, BSc OT (Wits), MSc OT (Wits), DHT (Pret) PhD (Wits) http://orcid.org/0000-000 I-8295-6329 Sessional Senior Lecturer, Department of Occupational Therapy, School of Therapeutic Sciences, Faculty of Health Sciences, University of the Witwatersrand

Patricia De Witt, Nat. Dip. OT (Pretoria); MSc OT (Wits); PhD (Wits) http://orcid.org/0000-0003-36 I2-0920

Sessional Adj Professor, Department of Occupational Therapy, School of Therapeutic Sciences, Faculty of Health Sciences, University of the Witwatersrand

Introduction: It is estimated that more than 200000 children under the age of I 4 years are living with human immunodeficiency virus (HIV) in South Africa. These children - including those on antiretroviral therapy - may present with neurocognitive delay and may find it difficult to participate in educational activities. This study aimed to determine the extent of the delay of visual motor integration (VMI) which correlates significantly with academic achievement in pre-school children with vertically transmitted HIV.

Method: This was a descriptive quantitative study in which 7 I children infected with HIV aged between 5 - 6 years, attending a HIV clinic in South Africa, were assessed using the Beery Developmental Test of Visual Motor Integration (DTVMI). The results were compared to the normative DTVMI scores, and correlated with health factors related to HIV such as CD4 count and socioeconomic factors such as attendance at crèche or preschool.

Results: Results confirm that a delay exists in VMI and visual perception (VP) in the at risk category. Mean scores on the motor coordination (MC) fell in the average range. Visual perception was the most affected in this sample with an average delay of between II and 17 months found. Visual perception also showed a moderate positive correlation with CD4 count, while VMI had a moderate relationship to attendance at crèche or preschool and mother's level of education.

Conclusion: This study confirms children with HIV are at risk for neurodevelopmental delay related to visual motor integration and visual perception, particularly in children with a low CD4 count. These aspects need to be assessed as part of routine neurodevelopmental monitoring.

Key words: Neurodevelopmental delay, HIV, preschool children, visual motor integration delay, visual perception delay, motor coordination

\section{INTRODUCTION}

Human Immunodeficiency Virus (HIV) causes a chronic condition that impacts the immune system resulting in infections and lifelong dependence on medication for survival. The growth and health as well as the economic and food security, psychosocial care, education and family composition of children with the virus are also affected'. The estimated number of children living with HIV globally in 2018 was 2.1 million $^{2}$ and at the time, it was estimated that 260000 of these children (aged 0 to 14) were living in South Africa, with only $58 \%$ on treatment ${ }^{2}$. According to the Joint United Nations Programme on HIV/AIDS (UNIAIDS) new infections in South African children declined from 25,000 in 2010 to 13,000 in $2017^{2}$. This could largely be due to the increase in the prevention of mother to child transmission (PMTCT) which reduced vertical HIV infections at birth from $3.6 \%$ in $201 \mathrm{I}$ to $\mathrm{I} .3 \%$ in $2017^{3}$. The National Strategic Plan for HIV, TB and STIs 2017-2022, aims to renew the focus on children with HIV and their families to reduce this rate even further ${ }^{2}$.

Although not yet optimal, the increase in the number of children in South Africa receiving antiretroviral therapy (ART) is due to the revision of the ART guidelines in 2013 and 2015 by the South African National Department of Health ${ }^{4}$. These revised guidelines specified that for children between the ages of 5 - 10 years, ART should commence either when symptomatic or when CD $4<500$ cells $/ \mu$ l regardless if the child was at WHO stage I (asymptomatic) WHO stage 2 (infections), WHO stage 3 (malnutrition and persistent infections) or WHO stage 4 (severe wasting and severe infections) ${ }^{5}$. Children younger than 5 years should receive ART regardless of their CD4 cell count or clinical staging and infants exposed at birth should be started on ART at 4-6 weeks of age ${ }^{4}$.

Ongoing care for children on ART should include monitoring their treatment response and adherence to ART as well as their developmental milestones ${ }^{6}$. Children whose viral load suppression is insufficient within the first 3 years of life including those on ART who are considered clinically stable, may present with neurocognitive decline ${ }^{7}$. This is due to HIV encephalopathy, associated with permanent neurocognitive deficits consistent with diffuse atrophy and bifrontal white matter abnormalities ${ }^{8,9}$.

In view of the deficits which may occur in relation to the degree of encephalopathy, children infected with HIV, even those on ART, often have difficulties in participating in school activities ${ }^{10}$. Occupational therapists need to monitor developmental milestones and address developmental delay in children with HIV, including visual motor integration (VMI), fine motor development and visual perceptual abilities required for formal education ${ }^{11-13}$. The relationship between $\mathrm{VMI}$ and academic achievement is not clear but VMI has been correlated significantly with academic ability, in relation to perceptual abilities needed for reading, writing, spelling and 
arithmetic ${ }^{14,15}$ and executive function ${ }^{16}$.

In South Africa the development of children with HIV must also be considered in view of the multiple vulnerabilities these children may be exposed to, including low socioeconomic status, poor nutrition, limited access to services and neglect ${ }^{17}$. Socioeconomic status which has been shown to be a factor related to VMI development with lower VMI scores reported for children from impoverished environments ${ }^{18,19}$. This factor was considered in this study where the VMI of preschool children with HIV receiving ART was established.

\section{LITERATURE REVIEW}

The efficacy of the treatment for HIV is determined by many factors such as the age of commencing treatment, the quantity of CD4 cells and the viral load ${ }^{20}$. While combination ART such as abacavir, lamivudine, opinavir, ritonavir, efavirenz and nevirapine with improved cerebrospinal fluid penetration ${ }^{4}$ have been associated with improved neurological function, a significant percentage of children still experience neurocognitive delay ${ }^{7}$. Since the CD4 count in children infected with HIV determines the extent of the spread of the HIV virus systemically, it also determines the degree of cognitive impairment ${ }^{21}$. Even when statistically significant changes in the CD4 percentage and viral load levels occur post ART initiation, deficits in visual perception and language scores in children over 3 years of age, remained unchanged ${ }^{22}$. This was confirmed by Knox, et al. ${ }^{23}$ who assessed I 58I preschool children in KwaZulu Natal and reported HIV positive children were more likely to have cognitive delay $(\mathrm{OR}=2.2,95 \% \mathrm{Cl}=1.2-3.9)^{23}$. A study by Puthanakit, et al. ${ }^{24}$ also found delayed neurocognitive developmental in specific areas such as IQ, memory, visual motor integration, fine motor skills and behaviour in 284 Thai and Cambodian HIV infected children aged I- 12 years. They suggested that ART should be initiated in infancy to address "irreversible but non progressive HIV brain insult even in the presence of successful ART when ART is initiated in older children."24:506. Boivin et al. ${ }^{25}$ confirmed the importance of early ART initiation on cognitive function. They found significantly higher scores on the Behavior Rating Inventory of Executive Function (BRIEF) and standardised Global Executive Composite (GEC) $(p=0.03)$ in preschool children who started ART before I year of age $^{25}$. However, Paul et al. ${ }^{26}$ found that even when children infected with HIV had been enrolled in the Pediatric Randomised Early versus Deferred Initiation programme they had significantly lower VMI scores $(p=0.04)$ than non-infected Cambodian children between the ages of 6-17 years. Cognitive function was also found be lower in children with CD4 cell counts $>350$ cells $/ \mathrm{mm}^{3}$ in Uganda ${ }^{27}$.

External factors such as high maternal viral loads ${ }^{28}$ and limited maternal education, prenatal drug exposure, poor household intellectual stimulation and malnutrition have been reported to impact negatively on neurocognitive development ${ }^{29}$. However, in children with HIV, neurocognitive deficits presenting from infancy across childhood development, including VMI, are more evident even when these factors, usually related to socioeconomic status, are controlled ${ }^{21}$. HIV has been known to "...have a greater effect on development than any other factors including poverty" $6: 120$ and it has been shown that providing support to combat the effects of a low socioeconomic status including nutritional deficits does also not significantly influence attainment of the fine motor skills required for VMI in HIV-infected children ${ }^{30}$.

Specific deficits in neurodevelopment in children with HIV, was confirmed by Fishkin et al. ${ }^{31}$ in a comparative study between 40 participants aged between 3 - 5 years and an equal number of non-infected controls, using the Wechsler Primary and Preschool Scale of Intelligence Revised (WPPSI-R). A significant difference between the groups was found on the Block Design subtest of the WPPSI-R. This subtest specifically requires processing speed, visual motor integration, sustained attention, motor speed and coordination, all functions associated with the frontal cortex and the parietal ${ }^{l o b e}{ }^{31}$. These findings were supported by Wang et al..$^{32}$ who found neurological deficits related to the Lateral Occipital Complex (LOC) network in 15 participants infected with HIV for less than one year, when compared to age-matched controls who were HIV negative. The participants underwent structural and functional MRIs and significant differences between the groups indicated deficits extending laterally towards the occipitotemporal region and dorsally into the posterior parietal region essential for VMI.

The impact of poor VMI development on the education occupational performance area must therefore be considered and emphasised in children with HIV. A significant relationship exists between visual analysis, fine motor, visual motor integration with handwriting, reading and mathematical performance in the early school years ${ }^{14,15}$. Visual-motor skills, or VMI is required for the integration of visual images of letters or shapes with a motor output and is an important pre-writing skill. These skills are multifaceted and are influenced by a number of factors such as pencil grip, fine motor skills, eye hand coordination, kinaesthesia, motor planning and visual perceptual skills. A good VMI performance is also a strong predictor of executive function related to self-regulation in the classroom ${ }^{16,33}$.

Since the extent of the delay in visual motor integration in HIV-infected children, particularly within the South African context, is largely unknown the objectives of the study were to determine:

$\leftrightarrow$ the extent of the delay of VMI in pre-school children who have vertically transmitted HIV,

$*$ the association between $\mathrm{VMI}$, visual perception and motor coordination scores and medical factors related to HIV,

$*$ the association between VMI, as the demographic and socioeconomic factors for children with HIV.

\section{METHOD}

A cross-sectional quantitative descriptive study in which no variables were manipulated, was undertaken to explore the visual motor integration, visual perception and motor coordination of 5 to 6 year old children infected with HIV who were receiving ART. Ideally a randomised clinical control study was desirable, but ethically this was not possible as the children who could potentially form the control group could not be tested to confirm if they were HIV positive. Socioeconomic status has been shown to be a factor related to Beery Developmental Test of Visual Motor Integration (DTVMI) scores ${ }^{34}$ but this is controversial as some authors found no delay related to low and middle socioeconomic status in preschool children ${ }^{34,35}$. However, in view of the absence of a control group, the socioeconomic background of the children living with HIV was assessed to ensure that the sample was homogeneous for this variable and the possible effect on the results could be discussed. Results on the DTVMI were also compared to normative results from other South African studies of children from different socioeconomic groups.

Data were collected at one point in time from a sample attending a paediatric outpatient clinic at an academic Hospital in Johannesburg that provides services to infants, children and adolescents with HIV from the greater Johannesburg region in South Africa. Patients attending the clinic are mostly from middle to low socioeconomic background. A sample of participants was selected using the following inclusion criteria:

$*$ ambulant children who were HIV positive through vertical transmission.

* children who were between the ages of 5 years 0 months and 5 years II months.

* children who had been receiving ART > I year.

The children were excluded from the study if they

$\star \quad$ were born prematurity with a gestation period of $<37$ weeks.

* had neurodevelopmental delay due to other causative factors such as neonatal hypoxic-ischaemia.

* had physical manifestations of encephalopathy such as seizures.

$\star$ had visual deficits. 
The estimated number participants required to be representative of the 1600 children attending the clinic according to Cochran's sample size formula with a margin of error of $5 \%$ or alpha of 0.05 for the study was $79^{36}$

Ethical clearance was obtained from the University of the Witwatersrand (MI 60876) and prior to commencing the assessment, the caregivers of the participants gave signed informed consent, with the assistance of a translator if needed. Verbal assent was also obtained from the child participants.

\section{Data Collection tools}

Demographic and Medical Information was collected using a questionnaire developed by the researcher for this study. The caregivers were also asked whether the participant had undergone an eye test and the outcome of the eye test to determine the possible functional impact of visual deficits on visual motor integration. Children with known visual deficits that could not be corrected with glasses were excluded from the study. However, a limitation of the study was that no further assessment of the children's visual function was completed so the presence of visual deficits may have affected the results. Medical information was obtained from the medical file and included details of ART, CD4 count, CD4\%, viral load and height/ weight of the participant.

\section{The Household Economic and Social Status Index (HESSI) II $^{37}$}

The variable of socioeconomic status was controlled for in this study and the HESSI II was used to collect information on the socioeconomic status of the participants on indicators of material wellbeing such as quality of housing and social capital such as mother's education and marital status ${ }^{37}$. The HESSI II is a self-report measure developed and standardised in Soweto, South Africa. It has been used in various studies to give an indication of the socioeconomic status of study participants ${ }^{22,38}$.

The Beery-Buktenica Developmental Test of VisualMotor Integration (Beery DTVMI) $6^{\text {th }}$ edition $^{39}$

The Beery DTVMI-6 is a standardised test and was used to assess the participants' VMI, with supplemental tests for visual perception (VP) and motor coordination (MC). This test has been widely used as a research tool. The Beery DTVMI -6 has good predictive validity ${ }^{39}$. The test's validity and reliability has been well-researched and is considered an effective measuring tool. The VMI, VP and MC tests have an overall average reliability score (inter-scorer, content and time sampling) of $0.92,0.9 \mathrm{I}$ and 0.90 respectively. The VMI, in particular, has high reliability and validity when compared with other assessment measures of perceptual-motor skills ${ }^{39}$.

Content validity was established through vigorous levels of item analyses and selection procedures over the years since its initial formulation in 1967. The results of the VMI test have been reported to show a statistically significant relationship with mathematical achievement test scores ${ }^{40}$. Therefore, apart from being used to determine the level of VMI, VP and MC skills, inferences on academic performance can be made, although this is to be done with caution. The Beery DTVMI- 6 has been found to be culture free $^{39}$ and is a commonly used research tool to determine the VMI, VP and MC of children internationally ${ }^{4}$. The Beery DTVMI- 6 was normed on a population of American children and has been shown to be valid for the assessment of children in South Africa ${ }^{42,43}$ with significant differences between the scores for preschool children in high to middle and low socioeconomic groups. Although children in middle and lower socioeconomic groups had scaled scores that reflected the normative score in the test manual ${ }^{34}$

\section{Research Procedure}

The data were collected for a period of eight months and assessments coincided with the participants' clinic appointment. The testing took place in a private room at the clinic and lasted approximately 30 minutes. The caregiver was asked to complete the demographics form and HESSI II with the help of a translator if necessary while the child was assessed by the researcher. The researcher collected the medical information from the participants' clinic records. The Beery DTVMI was administered according to the standard procedure with a research assistant, familiar with the test completing a direct translation of the written instructions in the manual.

\section{Data Analysis}

Demographic data, medical data and the data from the HESSI II were analysed using descriptive statistics including frequencies and ranges. The data from the Beery DTVMI were analysed using descriptive statistics including percentile and $z$ scores using the mean, standard deviation and confidence intervals. A chi-squared test was used to determine the significance of the results from the Beery DTVMI in comparison to the norms established by other authors in South Africa.

Correlations were also established between the medical factors and socioeconomic factors related to HIV and the VMI, VP and $\mathrm{MC} z$ scores $^{44}$.

\section{RESULTS}

A sample of 80 participants was initially selected for this study. During the screening process, 9 participants were found to be ineligible to participate as 3 clients received ART $<6$ months, I client was born prematurely, I client had a history of medical illnesses and 2 clients were above the age of 6 years and two participants were too ill to complete the assessment. This resulted in a final sample of 71 participants.

The demographic information revealed that $40.85 \%$ of the child participants were male and $59.15 \%$ were female with ages ranging from 5 years 0 months to 5 years II months. The home language of nearly half $(46 \%)$ of the participants was Zulu while between $3 \%$ and II $\%$ of participants spoke Tswana, Xhosa, Ndebele, Setswana followed by Northern Sotho and Shangaan at home. Other languages spoken included English, Afrikaans and French. Only I $4 \%$ of participants had not attended crèche, day care or a nursery/pre-primary school.

Socioeconomic data from the HESSI indicted all the participants came from a middle or low socioeconomic background. On average two adults and one child were reported living in each household. Thirty-six (5I\%) of the caregivers, who were all the child's mother, reported living with a partner while $35(48 \%)$ were single parents. Thirty-one (43\%) of the caregivers of the participants reported having completed three or five years of high school, with $32(45 \%)$ having post school training at technical college.

The caregivers' housing was located in the areas around the hospital, which mostly consisted of low-cost housing. While 23 $(32.39 \%)$ of the caregivers reported living in a house, $8 \%$ reported to sharing their home with other families. Fifteen $(21.13 \%)$ of the participants lived in a flat, while the remaining 27 (38\%) caregivers reported the family lived in a shack or single room/garage, which indicated a low socioeconomic status as $81 \%$ of these dwellings did not have indoor plumbing and some did not have electricity.

\section{Medical factors impacting on VMI}

The average length of time a participant had received ART was 3.5 years resulting in an average CD4\% of $31.41 \%$ and CD4 absolute count of I237.96XI0^6/L. (Table I):

Table I: Medical Information of participants $(n=7$ I)

\begin{tabular}{|l|l|}
\hline & Mean ( \pm SD) \\
\hline Years on ART & $3.53(1.58)$ \\
\hline CD4 absolute $\left(\times 10^{\wedge}\right.$ 6/L) & $1237.96 \pm 551.0$ \\
\hline CD4 (\%) & $31.41 \pm 8.8$ \\
\hline
\end{tabular}

The length of time on ART showed a positive moderate correlation with CD4\% $(r=0.5 \mathrm{I})$ indicating a positive association between ART and CD4\%. Interestingly a weak positive correla- 
tion $(r=0.38)$ existed between the length of time on ART with the CD4 count.

\section{Visual motor integration}

The results of the Beery DTVMI reveals that when compared to the USA norms ${ }^{39}$ the scores for VMI and VP fell in the "at risk" category below average with a mean $\mathrm{z}$ score of $-\mathrm{I} .13$ and -1.43 respectively. Motor coordination mean $\mathrm{z}$ scores fell in the average range at -0.86 . Visual perception achieved the lowest in this sample on the mean raw score and $20.5 \%$ of the $z$ scores falling -2SD below the mean (Figure $I$ on the left).

The Beery DTVMI scores were compared to the USA normative data since South African norms in means were not available for this age group., A significant difference existed between the $z$ scores of VMI $(p=0.00)$, VP $(p=0.00)$ and MC $(p=0.00)$ and the normal distribution of scores. These scores indicated that $13.6 \%$ of the sample presented with visual motor integration deficits with z scores below -2 SD, while $20.5 \%$ of the sample presented with deficits for visual perception in this range (Figure 2 on the left).

Table II (below) indicates that a delay exists in VMI, VP and MC in the sample in this study when compared to the normal distribution of scores for the United States with a scaled score of 10 (SD 3) ${ }^{34}$. When compared to scores reported for studies in South Africa ${ }^{29,36}$, the scores for the study sample are not significantly lower $(p=0.223)$ than the norms reported for South African pre-schoolers, but confirmed developmental delay. The mean scores are equivalent to the average scores for children of 4 years 6 months for VMI, children of 4 years for visual perception and children of 4 years 7 months for motor coordination. This indicates a deficit of I I months on the mean VMI raw scores and a deficit of 17 months for visual perception. The mean motor coordination scores show a deficit of 10 months.

\section{The relationship between medical factors and the Beery Developmental test of Visual Motor Integration}

Visual perception had a moderate positive significant correlation with the CD4 count and CD4 (\%) with weak correlation between these variables and the $\mathrm{VMI}$ and $\mathrm{MC}$ scores.

There were weak non-significant correlations between the other medical factors and all the Beery DTVMI scores. (see Table III on page 28)

The relationship between demographic and socioeconomic factors and the Beery Developmental test of Visual Motor Integration

Only one demographic factor (attendance at crèche, day care or nursery/pre-primary school) and one socioeconomic factor (mother's level of education) assessed in this study, correlated moderately with the VMI z scores. All correlations for the VP and MC z scores were weak. (see Table IV on page 28)

Figure 2: Comparison of z scores for participants living with HIV and the normal distribution for $z$ scores on the Beery Developmental Test of Visual Motor Integration $(n=7 I)$

Table II: Comparison of Beery Developmental Test of Visual Motor Integration score to United States and South African norms

\begin{tabular}{|c|c|c|c|c|}
\hline & \multicolumn{2}{|c|}{ VMI-6 Study sample scaled scores $(n=71)$} & \multirow{2}{*}{$\begin{array}{l}\text { VMI-4 South African } \\
\text { sample middle to low SES } \\
\text { (Dunn et al. } .^{34}(\mathbf{n}=83) \\
\text { Mean (SD) }\end{array}$} & \multirow{2}{*}{$\begin{array}{l}\text { VMI } 6 \text { South African } \\
\text { preschool norms (Visser } \\
\left.\text { and } \mathrm{Nel}^{42}\right)(\mathrm{n}=68) \\
\text { Median-(range) }\end{array}$} \\
\hline & Mean (SD) & Median-(range) & & \\
\hline Visual motor integration & $6.64(2.37)$ & $6(2-12)$ & $10.5(3.80)$ & $9(4-14)$ \\
\hline Visual perception & $6.04(2.91)$ & $6(1-14)$ & & $8(1-17)$ \\
\hline Motor co-ordination & $7.62(3.06)$ & $7(1-13)$ & & $10(5-17)$ \\
\hline
\end{tabular}


Table III: Correlations between CD4 count, CD4\% and z scores on the Beery Developmental test of Visual Motor Integration $(\mathbf{n}=7 \mathrm{I})$

\begin{tabular}{|l|c|c|}
\hline & CD4 count & CD4 (\%) \\
\hline VMI z score & \multicolumn{2}{|c|}{$\mathbf{r}$} \\
\hline VP z score & 0.00 & 0.12 \\
\hline MC z score & $\mathbf{0 . 5 6 *}$ & $\mathbf{0 . 4 6 *}$ \\
\hline Significance $\mathrm{p} \leq 0.05^{*}$ & 0.27 & 0.27 \\
\hline
\end{tabular}

Table IV: Correlations between demographic and socioeconomic factors and z scores on the Beery Developmental test of Visual Motor Integration $(n=7 I)$

\begin{tabular}{|l|c|c|}
\hline & $\begin{array}{c}\text { Attendance at crèche, day care or } \\
\text { nursery/pre-primary school }\end{array}$ & Mothers level of education \\
\hline VMI z score & \multicolumn{2}{|c|}{$\mathbf{r}$} \\
\hline VP z score & $\mathbf{0 . 5 6 *}$ & $\mathbf{0 . 4 3 *}$ \\
\hline MC z score & 0.32 & -0.13 \\
\hline Significance $p \leq 0.05 *$ & 0.11 & 0.33 \\
\hline
\end{tabular}

\section{DISCUSSION}

The participants in this study came from a variety of cultural backgrounds and had received ART for between one and five years. None of the participants started ART before the age of I year but there was only a weak correlation between the length of time on ART and their CD4 scores evidenced by the relatively high CD4 count and CD4\% scores found. These scores determine the extent of the spread of the virus systemically and the degree of cognitive impairment ${ }^{21}$. A stronger CD4 count will also decrease the likelihood of opportunistic infections that can negatively impact the visual motor area ${ }^{29,45}$.

Even with the initiation of ART, some neurocognitive delay occurred in this sample with a low average score on at least one of the Beery DTVMI tests. However, this was in line with South African studies linking lower and middle economic status to lower VMI scores ${ }^{34}$. The extent of the delay was not significant when the sample scores were compared to norms for typical South African pre-schoolers and those from low and middle socioeconomic contexts. Socioeconomic status in particular maternal education and the attendance at crèche/pre-primary school were shown to positively correlate with VMI scores in this study and are known to influence neurodevelopment in general ${ }^{29}$. Better maternal education and attendance at preschool provide a child with better metacognitive strategies for problem-solving tasks and feedback that is appropriate to the child's level of success in VMI tasks ${ }^{46}$.

However, in this study socioeconomic status was less of a mediating variable than the impact of $\mathrm{HIV}^{23}$ even when other factors such as poverty were considered ${ }^{24}$. This supports research studies that neurocognitive deficits, particularly visual perceptual deficits exist in children infected with HIV and receiving ART ${ }^{24,29}$ and that HIV is a major determinant of neurodevelopmental delay'.

HIV causes a delay in visual perception and visual motor integration ${ }^{26,32}$. Both the VMI and VP scores in this study were below average, indicating the presences of neurological deficits in the LOC and parietal lobes as reported by Wang et al. ${ }^{32}$. The parietal cortex, responsible for integrating and processing somatosensory visual, information, plays a role in producing planned movements required for $\mathrm{VMI}^{47}$. Motor coordination scoring was a low average. The findings for $M C$ in this study reflect those reported by Fraga-Sousa et al. ${ }^{48}$ that fine motor function in 5 year old children with HIV is relatively intact and not significantly different from that of typical children ${ }^{48}$. It must be noted that the scores obtained for VMI, VP and MC in this study reflect the findings of other studies in South Africa using the Beery DTVMI, In all the studies the participants obtained the lowest scores for VP and the highest scores for $M C$ when compared to normative data from the test manual ${ }^{18,34,42,43,49}$. Therefore, delay in VMI, VP and $M C$ must be interpreted accordingly when determining the real delay for this South African sample of HIV positive children.

It is important therefore that the delays in visual perception in children with HIV are addressed as it is necessary for visual information analysis to explore the environment and gain mastery in educational activities ${ }^{50,51}$. Visual perception and visual motor integration are integral to the development of prewriting and handwriting skills, as well as behaviour reflecting self-regulation which are necessary for achievement in the classroom ${ }^{16,50}$.

\section{Limitations of the study}

A possible limitation is the small sample size which was only representative of the population with a $10 \%$ margin of error ${ }^{36}$. When analysing the socioeconomic levels of the study participants, a composite score of the HESSI II could not be obtained as not all participants completed all sections however, from the information completed a socioeconomic level could be concluded in all cases. The visual function of the children was not assessed and only based on a report from the caregivers.

\section{CONCLUSION}

The results from this study supported the conclusion that HIV is a major determinant of neurodevelopmental delay with child participants at risk for delay on the Beery VMI test. There was an association the prevalence of visual perception delay and the level of the CD4 count and CD4\% which has clinical and research implications on the extent of the delay. The middle to low socioeconomic status of the participants was a mediating factor even when compared to other standardisation studies of the Beery DTVMI on a South African population, with participants in this study had lower scores confirming that HIV is a major determinant of dysfunction in neurodevelopment.

It is recommended that a combination of ART and neurodevelopmental occupational therapy intervention is necessary, particularly at a preschool and the early school level for children with HIV and that VMI, VC and VP should be assessed as part of routine neurodevelopmental monitoring in these children. 


\section{REFERENCES}

I. Cornia GA. AIDS, public policy and child well-being. UNICEF Innocenti Research Centre, 2007.

2. Joint United Nations Programme on HIV/AIDS. UNAIDS: South Africa. https://www.unaids.org/en/regionscountries/countries/southafrica (2018, accessed 12 January 2019).

3. The South African National AIDS Council. Let our actions count: Reflections on NSP 2012-2016 and moving forward to NSP 2017-2022, http://nsp.sanac.org.za/wp-content/uploads/20 I 7/0 I/Final-NSPDocument.pdf (2017, accessed 23 July 2017).

4. Department of Health. National Consolidated Guidelines for the Prevention of Mother-to-Child Transmission of HIV (PMTCT) and the Management of HIV in Children, Adolescents and Adults. 2015. Pretoria: National Department of Health.

5. World Health Organization. WHO case definitions of HIV for surveillance and revised clinical staging and immunological classification of HIV-related disease in adults and children. 2007.

6. Baillieu $\mathrm{N}$ and Potterton J. The extent of delay of language, motor, and cognitive development in HIV-positive infants. Journal of neurologic physical therapy. JNPT. 2008; 32: I I 8- I 2 I . 2008/ I I/04. DOI: https://doi.org/10.1097/NPT.0b013e3181846232.

7. Weber V, Radeloff D, Reimers B, et al. Neurocognitive development in HIV-positive children is correlated with plasma viral loads in early childhood. Medicine. 2017; 96: e6867. https://doi.org//0.1097/MD.0000000000006867.

8. Uban K, Herting M, Williams P, et al. White matter microstructure among youth with perinatally acquired HIV is associated with disease severity. AIDS. 2015; 29: 1035-1044. https://doi.org/10.1097/QAD.0000000000000648.

9. Shanbhag MC, Rutstein RM, Zaoutis T, et al. Neurocognitive functioning in pediatric human immunodeficiency virus infection: effects of combined therapy. Archives of Pediatrics \& Adolescent Medicine. 2005; I59: 65I-656.

https://doi.org/10.1001/archpedi.159.7.65I.

10. Puthanakit T, Aurpibul L, Louthrenoo O, et al. Poor cognitive functioning of school-aged children in Thailand with perinatally acquired HIV infection taking antiretroviral therapy. AIDS, Patient care and STDs. 2010; 24: |4l-146. https://doi.org/10.1089/apc.2009.03/4.

II. Stevens M, Kirsh B and Nixon SA. Rehabilitation interventions for children living with HIV: a scoping review. Disability and Rehabilitation. 20।4; 36: 865-874.

https://doi.org/10.3109/09638288.2013.82। I 84

12. Meissner RJ, Ferguson J, Otto C, et al. A play-informed, caregiverimplemented, home-based intervention for HIV-positive children and their families living in low-income conditions in South Africa. World Federation of Occupational Therapists Bulletin. 2017; 73: 83-87. https://doi.org/10.1080/|4473828.2017.1375068

13. Musindo O, Bangirana P, Kigamwa P, et al. Neurocognitive functioning of HIV positive children attending the comprehensive care clinic at Kenyatta national hospital: exploring neurocognitive deficits and psychosocial risk factors. AIDS Care. 2018; 30: 618-622. https://doi.org/10.1080/09540121.2018.1426829.

14. Clark GJ. The relationship between handwriting, reading, fine motor and visual-motor skills in kindergarteners. 2010.Unpublished dissertaion. University of Iwoa.

15. Carlson AG, Rowe E and Curby TW. Disentangling fine motor skills' relations to academic achievement: the relative contributions of visual-spatial integration and visual-motor coordination. The Journal of Genetic Psychology. 2013; 174: 514-533. https:// doi.org/10.1080/00221325.2012.717122

16. Kim H, Byers Al, Cameron CE, et al. Unique contributions of attentional control and visuomotor integration on concurrent teacherreported classroom functioning in early elementary students. Early Childhood Research Quarterly. 2016; 36: 379-390. https://doi.org/10.1016/j.ecresq.2016.01.018.

17. Pillay J. Early Education of orphans and vulnerable children: A crucial aspect for social justice and African development. Koers. 2018; 83: $1-12$.

https://dx.doi.org/10.19108/koers.83.1.2335

18. van Heerden C, de Kock N, Larsen K, et al. Visual motor integration in children living in childcare institutions in Gauteng. South African Journal of Occupational Therapy. 201 I; 41 : 38-43.
19. Pienaar A, Barhorst R and Twisk J. Relationships between academic performance, SES school type and perceptual-motor skills in first grade South African learners: NW-CHILD study. Child: Care, Health and Development. 2014; 40: 370-378. https://doi.org/I0. I I I I/cch. 12059.

20. Resino S, Resino R, Micheloud D, et al. Long-term effects of highly active antiretroviral therapy in pretreated, vertically HIV type I-infected children: 6 years of follow-up. Clinical Infectious Diseases. 2006; 42: 862-869. https://doi.org/10.1086/5004I2.

2I. Burns S, Hernandez-Reif M and Jessee P. A review of pediatric HIV effects on neurocognitive development. Issues in Comprehensive Pediatric Nursing. 2008; 31 : 107-121. https://doi.org/10.1080/01460860802272870

22. Potterton J, Stewart A, Cooper P, et al. The effect of a basic home stimulation programme on the development of young children infected with HIV. Developmental Medicine \& Child Neurology. 20 I0; 52: 547-55I. https://dx.doi.org/ I0. I I I I/j. I469-8749.2009.03534.x.

23. Knox J, Arpadi SM, Kauchali S, et al. Screening for developmental disabilities in HIV positive and HIV negative children in South Africa: Results from the Asenze Study. PloS one. 2018; 13: e0199860. https://doi.org/10.1371/journal.pone.0199860.

24. Puthanakit T, Ananworanich J, Vonthanak S, et al. Cognitive function and neurodevelopmental outcomes in HIV-infected children older than I year of age randomized to early versus deferred antiretroviral therapy: the PREDICT neurodevelopmental study. The Pediatric Infectious Disease Journal. 20I3; 32: 501 . https://dx.doi.org/10.1097\%2FINF.0b0 13e31827fb I9d.

25. Boivin MJ, Barlow-Mosha L, Chernoff MC, et al. Neuropsychological performance in African children with HIV enrolled in a multisite antiretroviral clinical trial. AIDS (London, England). 2018; 32: I89-204. https://dx.doi.org/10.1097\%2FQAD.0000000000001683.

26. Paul R, Prasitsuebsai W, Jahanshad N, et al. Structural Neuroimaging and Neuropsychologic Signatures in Children With Vertically Acquired HIV. The Pediatric Infectious Disease Journal. 2018; 37 : 662-668. https://doi.org//0.1097/INF.000000000000I852.

27. Ruel TD, Boivin MJ, Boal HE, et al. Neurocognitive and motor deficits in HIV-infected Ugandan children with high CD4 cell counts. Clinical Infectious Diseases. 2012; 54: 1001-1009. https://doi.org/10.1093/cid/cirl 037.

28. Mitchell W. Neurological and developmental effects of HIV and AIDS in children and adolescents. Mental Retardation and Developmental Disabilities Research Reviews. 2001; 7: 21 I-216. https://doi.org/10.1002/mrdd. 1029.

29. Smith L, Adnams $C$ and Eley B. Neurological and neurocognitive function of HIV-infected children commenced on antiretroviral therapy. South African Journal of Child Health. 2008; 2: I08-II 3.

30. Nkirote L, Muchiri EM and Kombe Y. Effects of nutrition support on attainment of motor skills milestones in children receiving antiretroviral therapy (ART) in selected slums of Nairobi, Kenya. Journal of Health, Medicine and Nursing. 2019; 4: 30-49.

31. Fishkin PE, Armstrong FD, Routh DK, et al. Brief report: relationship between HIV infection and WPPSI-R performance in preschool-age children. Journal of pediatric psychology. 2000; 25: 347-35 I. 2000/07/06. https://doi.org/10.1093/jpepsy/25.5.347.

32. Wang $\mathrm{X}$, Foryt $\mathrm{P}$, Ochs $\mathrm{R}$, et al. Abnormalities in resting-state functional connectivity in early human immunodeficiency virus infection. Brain Connectivity. 201 I; I: 207-217. https://doi.org/10.1089/brain.201 1.0016.

33. Verdine BN, Irwin CM, Golinkoff RM, et al. Contributions of Executive Function and Spatial Skills to Preschool Mathematics Achievement. Journal of Experimental Child Psychology. 2014; I26: 37-5 I. https://dx.doi.org/10.1016\%2Fj.jecp.2014.02.012.

34. Dunn $M$, Loxton $\mathrm{H}$ and Naidoo A. Correlations of scores on the developmental test of visual-motor integration and copying test in a South African multi-ethnic preschool sample. Perceptual and Motor skills. 2006; 103: 951-958. https://doi.org/10.2466/pms. 103.3.95I-958.

35. Vally Z. The Beery Developmental Test of Visual Motor Integration: A Reliability Study. Unpublished research report. University of Stellenbosch, Stellenbosch: 2006.

36. Bartlett J, Kotrlik J and Higgins C. Organizational research: Determining appropriate sample size in survey research appropriate 
sample size in survey research. Information Technology, Learning, and Performance Journal. 200I; 19: 43.

37. Barbarin OA and Khomo N. Indicators of economic status and social capital in South African townships: What do they reveal about the material and social conditions in families of poor children? Childhood (Copenhagen, Denmark). 1997; 4: 193-222.

38. Barbarin OA and Richter L. Economic Status, Community Danger and Psychological Problems among South African Children. Childhood (Copenhagen, Denmark). 200I; 8: II 5-I33. https://doi.org/10.II77/090756820I00800I007.

39. Beery KE and Beery NA. The Beery-Buktenica Developmental Test of Visual-Motor Integration (Beery VMI) with Supplemental Developmental Tests of Visual Perception and Motor Coordination and stepping stones age norms: Administration, scoring and teaching manual. Minneapolis, Pearson, 2010.

40. Taylor Kulp M. Relationship between visual motor integration skill and academic performance in kindergarten through third grade. Optometry and Vision Science. 1999; 76: 159-163.

4I. Sortor JM and Kulp MT. Are the results of the Beery-Buktenica Developmental Test of Visual-Motor Integration and its subtests related to achievement test scores? Optometry and Vision Science. 2003; 80: 758-763.

42. Visser $M$ and Nel M. Visual perception of five-year-old Afrikaansspeaking children in Bloemfontein using the Beery VMI-6, DTVP-3 and TVPS-3: A follow up study of their English-speaking counterparts in Bloemfontein. South African Journal of Occupational Therapy. 20I8; 48: 31-43. http://dx.doi.org//0.17/59/2310-3833/2017/vol48n3a6.

43. Harris M. The validity of standardised visual perceptual tests in identifying specific learning disabilities in children from Gauteng Province, South Africa. In: Presentation: 35th Occupational Therapy Association (OTASA) Congress. 2016: I4-16.

44. Chen PY and Popovich PM. Correlation: Parametric and nonparametric measures. SAGE Publications, Inc, 2002.

45. Pizzo PA and Wilfert CM. Pediatric AIDS: the challenge of HIV infection in infants, children, and adolescents. Philadelphia: Lippincott Williams and Wilkins, 1994.

46. Carr A and Pike A. Maternal scaffolding behavior: links with parenting style and maternal education. Developmental psychology. 2012; 48: 543. https://psycnet.apa.org/doi/I0.1037/a0025888.

47. Stevens $A$ and Bernier R. Visual-Motor Function. Encyclopedia of Autism Spectrum Disorders. 2013: 3318-3319.

48. Fraga-Sousa GA, Rodrigues MCR, Pereira MG, et al. Motor performance of HIV-positive children. Fisioterapia em Movimento. 2016; 29: $61-70$ http://dx.doi.org//0.1590/0103-5150.029.00I.AO06.

49. Rens $Z$. The standardization of the Beery-Buktenica developmental test of visual-motor integration with supplemental developmental tests of visual perception and motor coordination (revised, 1997) on an Eastern Cape population aged 7 years 0 months to 7 years 3 months. University of the Witwatersrand, Johannesburg, 2009.

50. Case-Smith J and O'Brien JC. Occupational therapy for children and adolescents-E-Book. Elsevier Health Sciences, 2013.

5I. Kramer P. Frames of reference for pediatric occupational therapy. Philadelphia: Lippincott Williams \& Wilkins, 2018.

\section{AUTHOR'S ROLES}

- Ramona Odejayi - Postgraduate MSC OT study

- Denise Franzsen- Supervisor of study

- Patricia de Witt - conceptualisation and editing of article

Corresponding Author

\section{Ramona Odejayi}

Email: ramona.odejayi@gmail.com 